\title{
COUNTING SHORT CYCLES OF (C,D)-REGULAR BIPARTITE GRAPHS
}

\author{
M. ALINEJAD AND K. KHASHYARMANESH
}

\begin{abstract}
Recently, working on the Tanner graph which represents a low density parity check (LDPC) code becomes an interesting research subject. Finding the number of short cycles of Tanner graphs motivated Blake and Lin to investigate the multiplicity of cycles of length girth in bi-regular bipartite graphs, by using the spectrum and degree distribution of the graph. Although there were many algorithms to find the number of cycles, they preferred to investigate in a computational way. Dehghan and Banihashemi counted the number of cycles of length $g+2$ and $g+4$, where $G$ is a bi-regular bipartite graph and $g$ is the length of the girth $G$. But they just proposed a descriptive technique to compute the multiplicity of cycles of length less than $2 g$ for biregular bipartite graphs. In this paper, we find the number of cycles of length less than $2 g$ by using spectrum and degree distribution of bi-regular bipartite graphs such that the formula depends only on the partitions of positive integers and the number of closed cycle-free walks from a variable (resp. check) vertex in $\mathcal{B}_{c, d}$ and $\mathcal{T}_{c, d}$ (resp. $\mathcal{T}_{d, c}$ ), which are known.
\end{abstract}

\section{INTRODUCTION}

Low density parity check (LDPC) codes are linear codes with error performance near to the Shannon limit can represent as Tanner graphs, which proposed the first time by Michael Tanner. It is shown that the structure of Tanner graphs, in particular, distribution and number of short cycles, affect the error efficiency of LDPC codes (see [8, 9, 13, 14]). Performance of the LDPC codes persuade many researchers to investigate on cycles of Tanner graphs. In [2, 11], are shown that for finding LDPC codes with a good performance, we should focus on the graphs that the number of short cycles is not so many. It is proved that regularity of a graph has a significant impact on LDPC codes [12. Dehghan and Banihashemi in [5], studied cycle distribution of random bipartite graphs.

Counting the number of cycles in a general graph is known to be NP-hrad [6]. The complexity of the problem led the researcher to use recursion methods and algorithms to compute the number of cycles in bipartite graphs. For instance, in [7, Halford and Chugg proposed a recursive algorithm for counting the cycles of length at most $g+6$. In [10], Karimi and Banihashemi presented an algorithm to compute the number of cycles of length less than $g$. Recently, Blake and Lin suggested a new way, independent from algorithms and complicated methods, to compute the number of cycles by using spectrum and degree distribution of bipartite graphs [1].

Key words and phrases. $(c, d)$-regular graph, bipartitel graph, closed walks, cycle-free walk. 
For a given graph $\mathrm{G}$, the adjacency matrix $A=\left[a_{i j}\right]$ of $G$ is defined such that $a_{i j}=1$, if $i j \in E(G)$, and $a_{i j}=0$, if $i j \notin E(G)$. The spectrum of a graph $G$, denoted by $\left\{\lambda_{i}\right\}$, is the multiset of eigenvalues of adjacency matrix $A$. Since there exists a close relationship between the number of walks of arbitrary length and powers of matrix $A$, the spectrum of $G$ is more useful to find the number of cycles. Blake and Lin in [1] found the number of short cycles of length $g$ in bi-regular bipartite graphs without using complicated algorithms. They were hoping this new method guided the researchers to find the number of cycles of length greater than $g$. In [4], Dehghan and Banihashemi determined the exact number of cycles of length $g+2$ and $g+4$ in a bi-regular bipartite graph. In addition, by contradiction examples, they showed that the spectrum and degree distribution conditions are not enough to find the number of cycles of length $i$ for a bi-regular bipartite graph, where $i \geq 2 g$. They also mentioned some facts for the number of cycles of length less than $2 g$, but they did not proposed a formula to compute the number of cycles. By using the eigenvalues and degree sequence of bi-regular bipartite graphs, we present a new way to enumerate the number of cycles of length less than $2 g$ in bi-regular bipartite graphs.

In Section 2 of the paper, we present some definitions and preliminaries which we need through this paper. In Section 3, by using the partitions of positive integer numbers, we find the number of closed walks with a cycle in bi-regular bipartite graphs in which initial vertex is in cycle. In Section 4 , similar to section 3 , we investigate the number of closed walks that consist a cycle and initial vertex is out of cycle. Finally, from the results of sections 3 and 4, we determine the number of closed walks with cycle. Since the number of closed cycle-free walks in bi-regular bipartite graphs specified in [1], we can express the number of cycles of length less than $2 g$.

\section{Preliminaries and Notations}

For any graph $G$, we denote the set of all vertices and edges of $G$ by $V(G)$ and $E(G)$, respectively. For two vertices $u, v \in V(G)$, we denote $u \sim v$ or $u v$ for brevity, if $u$ and $v$ are adjacent. The degree of a vertex $v \in V(G)$, denoted by $d(v)$, is the number of adjacent vertices of $v$. A walk $\mathcal{W}$ is a sequence of the vertices $v_{1}, v_{2}, \ldots, v_{k+1}$ such that $v_{j} v_{j+1} \in E(G)$, for $1 \leq j \leq k$. In this case, $v_{j}$ is called the $j$-th vertex of $\mathcal{W}$ and the length of $\mathcal{W}$ is defined as the number of edges of $\mathcal{W}$ and is denoted by $\ell(\mathcal{W})$. We call $v_{1}$ and $v_{k+1}$ the initial and terminal vertex of $\mathcal{W}$, respectively. For integers $j$ and $s$, a walk $\mathcal{W}^{\prime}=v_{j}, \ldots, v_{v_{j+s}}$ is a subwalk of $\mathcal{W}=v_{1}, v_{2}, \ldots, v_{k+1}$, if $1 \leq j<k+1$ and $1<j+s \leq k+1$. A walk is called a closed walk if the initial and terminal vertex are the same. A closed cycle-free walk is a closed walk with no cycles. A closed walk $\mathcal{W}$ which is not a cycle is called a closed walk with cycle, if the induced subgraph on the edges of the closed walk has at least one cycle. For brevity, we denote the closed walk with cycle by CWWC. If the vertices of a walk are distinct, then a walk or closed walk is called path and cycle, respectively. For $u, v \in V(G), d(u, v)$ denotes the length of the shortest path between $u$ and $v$. If there is no path between $u$ and $v$, then we 
define $d(u, v)=\infty$. For a graph $G$, length of shortest cycle is called girth, and is denoted by $g$. For $j \geq g$, the number of cycles of length $j$ is denoted by $N_{j}$.

Graph $G$ is called bipartite, if $V(G)$ can be partitioned into two sets $U$ and $V$ such that if $u v \in E(G)$, then $u$ and $v$ belong to different sets. A graph $G$ is called nonbipartite, if $G$ is not bipartite. If the degree of vertices $U$ and $V$ are $c$ and $d$, respectively, then $G$ is called $(c, d)$-regular bipartite graph, and is denoted by $\mathcal{B}_{c, d}$. In this case, we assume that $|U|=n$ and $|V|=m$. For a bipartite graph $G=U \cup V$, the $m \times n$ parity check matrix $H(G)=\left[h_{i j}\right]$ defined in which $h_{i j}=1$, if $i j \in E(G)$, and $h_{i j}=0$, otherwise. Clearly, $H(G)$ constructs a linear code $C(G)$. In this case, $G$ is called the Tanner graph of $C(G)$. We denote $b_{c, 2 k}$ and $a_{c, 2 k}$ (resp. $b_{d, 2 k}$ and $a_{d, 2 k}$ ) as the number of closed cycle-free and return once closed cycle-free walks of length $2 k$ with initial vertex of degree $c$ in $\mathcal{B}_{c, d}$ (resp. $\left.d\right)$. For $k=0$, we assume that $b_{c, 0}=b_{d, 0}=1$.

Graph $G$ is connected, if there is a path between each two vertices of $G$. A connected graph with no cycle is called tree. For graph $\mathcal{B}_{c, d}$, the related tree of $\mathcal{B}_{c, d}$, denoted by $\mathcal{T}_{c, d}\left(\right.$ resp. $\left.\mathcal{T}_{d, c}\right)$, is defined as the rooted tree with root vertex of degree $c-1$ (resp. $d-1$ ) and vertices of consecutive levels have alternating degrees $d$ (resp. $c$ ) and $c$ (resp. $d$ ). In addition, we denote $t_{c, 2 k}$ and $s_{c, 2 k}$ (resp. $t_{d, 2 k}$ and $\left.s_{d, 2 k}\right)$ as the number of closed cycle-free and return once closed cycle-free walks of length $2 k$ in $\mathcal{T}_{c, d}\left(\right.$ resp. $\mathcal{T}_{d, c}$ ) with initial vertex of degree $c$ (resp. $d$ ). For $k=0$, we assume that $t_{c, 0}=t_{d, 0}=1$.

Clearly, the adjacency matrix $A$ is real and symmetric, and so the eigenvalues of $G$ are real. Moreover, it is known that if $\left\{\lambda_{i}\right\}$ is the spectrum of $G$, then $\left\{\lambda_{i}^{k}\right\}$ is the eigenvalue of $A^{k}$, for a positive integer $k$. For a matrix $A, \operatorname{tr}(A)$ is defined as the summation of diagonal entries of $A$. The following proposition play an important role in the enumerating the number of cycles.

Proposition 2.1. [3, Proposition 1.3.4] If $A$ is the adjacency matrix of a graph, then $(i, j)$-entry $a_{i j}^{k}$ of the matrix $A^{k}$ is equal to the number of walks of length $k$ that start at vertex $i$ and end at vertex $j$.

Since $\operatorname{tr}(A)$ is equal to the summation of eigenvalues of $A$, Proposition 2.1 implies that the number of closed walks of length $k$ equals the summation of eigenvalues of $A^{k}$. The Following theorem shows the difference of the spectrum of bipartite and the spectrum nonbipartite graphs.

Theorem 2.2. [3, Theorem 3.2.3] A graph $G$ is bipartite if and only if its spectrum is symmetric with respect to the origin.

Now, we express the following theorem from [4], which shows the number of cycles of length $i$, where $i<2 g$.

Theorem 2.3. [4, Theorem 1] For a $(c, d)$-regular bipartite graph $\mathcal{B}_{c, d}$, the number of cycles of length $i$ is equal to:

$$
N_{i}=\left[\sum_{j=1}^{|V(G)|} \lambda_{j}^{i}-\Omega_{i}\left(c, d, \mathcal{B}_{c, d}\right)-\Psi_{i}\left(c, d, \mathcal{B}_{c, d}\right)\right] / 2 i,
$$


where $\left\{\lambda_{j}^{i}\right\}$ is the spectrum of $\mathcal{B}_{c, d}$, and $\Omega_{i}\left(c, d, \mathcal{B}_{c, d}\right)$ and $\Psi_{i}\left(c, d, \mathcal{B}_{c, d}\right)$ are the number of closed cycle-free walks of length $i$ and closed walks with cycle of length $i$ in $G$, respectively.

In [1], Blake and Lin have already found the value $\Omega_{i}\left(c, d, \mathcal{B}_{c, d}\right)$ and showed that

$$
\Omega_{i}\left(c, d, \mathcal{B}_{c, d}\right)=n b_{c, i}+m b_{d, i} .
$$

In this work, we compute the number of closed walks of length $i+2 k, 1 \leq$ $k<g-\frac{i}{2}$, which contain a cycle of length $i, i<2 g$. For each walk of $\mathcal{B}_{c, d}$ we can consider a direction. Let $\mathcal{W}$ be an arbitrary closed walk with cycle of length $i$. By passing the walk sequence of $\mathcal{W}$, if we traverse clockwise in the cycle, then we define the direction of $\mathcal{W}$ is clockwise. Otherwise, we define the direction of $\mathcal{W}$ is counterclockwise. A CWWC walk with direction counterclockwise is denoted by $C W D C C$. Now, suppose that $\mathcal{C}$ is a cycle of length $i$ with vertices $v_{j}, 0 \leq j \leq i-1$ (see Fig. 1). Throughout this paper, indices of vertices of $\mathcal{C}$ are taken modulo $i$ and $d\left(v_{0}\right)=c$.

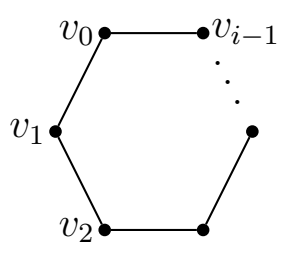

Fig 1.

\section{Closed walks in $(c, d)$-Regular Graphs With initial Vertex in CYCLE}

Definition 3.1. For integer $k$, where $1 \leq k<g-\frac{i}{2}$, the set of closed walks of length $i+2 k$ with cycle of length $i$ such that the initial vertex is in cycle or out of cycle is denoted by $\Phi(i, 2 k)$ or $\Lambda(i, 2 k)$, respectively. Therefore,

$$
\Psi_{i}\left(c, d, \mathcal{B}_{c, d}\right)=\sum_{k_{0}+k_{1}=g-\frac{i}{2}-1}\left(\left|\Phi\left(g+2 k_{0}, 2 k_{1}\right)\right|+\left|\Lambda\left(g+2 k_{0}, 2 k_{1}\right)\right|\right) .
$$

Definition 3.2. Suppose that $\mathcal{W}$ is a CWDCC with walk sequence $u_{1}, \ldots, u_{k+1}$. For a positive integer $j$ less than $k+1$, a closed cycle-free walk $\mathcal{W}^{\prime}$ with initial vertex $u_{j}$ is called backward respect to the $\mathcal{W}$, if $u_{j} u_{j+1} \notin E\left(\mathcal{W}^{\prime}\right)$. The walks $\mathcal{W}^{*}$ and $\mathcal{W}^{* *}$ are defined as the CWDCC of Fig. 1 and Fig. 2 with starting vertex $v_{0}$ and $z_{0}$, respectively.

In this section, we investigate the number of walks of $\Phi(i, 2 k)$ that consists cycle $\mathcal{C}$. We first determine the number of CWDCCs of $\Phi(i, 2 k)$ with cycle $\mathcal{C}$ and initial vertex $v_{0}$, denoted $\Phi_{v_{0}}(i, 2 k)$.

Remark 3.3. Suppose that $\mathcal{W} \in \Phi_{v_{0}}(i, 2 k)$. Then represent $\mathcal{W}$ as a sequence of vertices. Denote the first $v_{0}$ which appears in the sequence by $v_{0}^{(1)}$, and the first 
$v_{j}$ that appears after $v_{j-1}^{(1)}$ denote by $v_{j}^{(1)}$, for every $j, 0<j \leq i$. Since $\mathcal{W}$ is a CWDCC, we deduce that the vertex before $v_{j}^{(1)}$ in the sequence is $v_{j-1}$, where $0<j \leq i$. Hence, we can represent $\mathcal{W}$ as follow:

$$
v_{0}^{(1)}, \ldots, v_{0}, v_{1}^{(1)}, \ldots, v_{1}, v_{2}^{(1)}, \ldots, v_{i-1}, v_{i}^{(1)}, \ldots, v_{0} .
$$

Now, define closed subwalk $\mathcal{W}_{j}$ of $\mathcal{W}$ with initial vertex $v_{j}^{(1)}$ and terminal vertex $v_{j+1}^{(1)}$, for each $j$ with $0 \leq j \leq i-1$. Moreover, the walk with initial vertex $v_{i}^{(1)}$ and terminal vertex $v_{0}$ (The last vertex of $\left.\mathcal{W}\right)$ is denoted by $\mathcal{W}_{i}$. Thus, we can represent $\mathcal{W}$ uniquely as follow:

$$
\mathcal{W}=\mathcal{W}_{0} \mathcal{W}_{1} \ldots \mathcal{W}_{i}
$$

Now, put $2 s_{i}^{\prime}=\ell\left(\mathcal{W}_{i}\right)$, and $2 s_{j}^{\prime}=\ell\left(\mathcal{W}_{j}\right)-1$, for each $j$ with $0 \leq j \leq i$. By simple computing, we have

$$
\begin{gathered}
s_{0}^{\prime}+\ldots+s_{i}^{\prime}=\frac{1}{2}\left(\sum_{j=0}^{i} \ell\left(\mathcal{W}_{j}\right)-\sum_{j=0}^{i-1} 1\right) \\
=\frac{1}{2}(\ell(\mathcal{W})-i) \\
=\frac{1}{2}(i+2 k-i)=k .
\end{gathered}
$$

Definition 3.4. We denote $\Phi_{v_{0}}\left(2 s_{0}, \ldots, 2 s_{i}\right)$ for the walks $\mathcal{W} \in \Phi_{v_{0}}(i, 2 k)$ such that if $\mathcal{W}$ represent uniquely as $\mathcal{W}=\mathcal{W}_{0} \mathcal{W}_{1} \cdots \mathcal{W}_{i}$, then $2 s_{j}=\ell\left(\mathcal{W}_{j}\right)-1$ for $0 \leq j \leq i-1$, and $2 s_{i}=\ell\left(\mathcal{W}_{i}\right)$.

By the Remark 2.1, we have following result.

Corollary 3.5. For a positive integer $k$ with $1 \leq k<g-\frac{i}{2}$, we have:

$$
\Phi_{v_{0}}(i, 2 k)=\bigcup_{s_{0}+\ldots+s_{i}=k} \Phi_{v_{0}}\left(2 s_{0}, \ldots, 2 s_{i}\right)
$$

It is easy to see that the number of CWDCCs with initial vertex $v_{2 j}, 0 \leq j \leq$ $\frac{i}{2}-1$, is equal. Similar result satisfies for $v_{2 j+1}$, where $0 \leq j \leq \frac{i}{2}-1$. Since the number of vertices of degree $c$ and $d$ are $\frac{i}{2}$ and each CWWC has two directions, we have following result.

Corollary 3.6. The number of walks of $\Phi(i, 2 k)$ with cycle $\mathcal{C}$ is equal to:

$$
i\left(\sum_{s_{0}+\ldots+s_{i}=k}\left|\Phi_{v_{0}}\left(2 s_{0}, \ldots, 2 s_{i}\right)\right|+\left|\Phi_{v_{1}}\left(2 s_{0}, \ldots, 2 s_{i}\right)\right|\right) .
$$

Lemma 3.7. If $s_{0}+\ldots+s_{i}=k$, then

$$
\left|\Phi_{v_{0}}\left(2 s_{0}, \ldots, 2 s_{i}\right)\right|=b_{c, 2 s_{i}} \prod_{j=0}^{\frac{i}{2}-1} t_{c, 2 s_{2 j}} t_{d, 2 s_{2 j+1}},
$$


and

$$
\left|\Phi_{v_{1}}\left(2 s_{0}, \ldots, 2 s_{i}\right)\right|=b_{d, 2 s_{i}} \prod_{j=0}^{\frac{i}{2}-1} t_{d, 2 s_{2 j}} t_{c, 2 s_{2 j+1}} .
$$

Proof. By the definition of $\Phi_{v_{0}}\left(2 s_{0}, \ldots, 2 s_{i}\right)$, we know that $2 s_{j}$ is the length of the closed cycle-free walk with initial vertex $v_{j}$ in which it is backward respect to $\mathcal{W}^{*}$, for each $j, 0 \leq j \leq i-1$. Hence, Dependes on whether the degree of $v_{j}$ is $c$ or $d$, the number of walks of length $2 s_{j}$ with this condition is equal to $t_{c, 2 s_{j}}$ or $t_{d, 2 s_{j}}$, respectively. On the other hand, $2 s_{i}$ is the length of closed cycle-free walks with cycle and initial vertex $v_{0}$. Hence, the number of these walks equals to $b_{c, 2 s_{i}}$ or $b_{d, 2 s_{i}}$. Similar result is satisfied for $\left|\Phi_{v_{1}}\left(2 s_{0}, \ldots, 2 s_{i}\right)\right|$.

Since we computed the value $|\Phi(i, 2 k)|$ for specific cycle $\mathcal{C}$ and we have $N_{i}$ cycles of length $i$, we have following result.

Corollary 3.8. For integer $k, 1 \leq k<g-\frac{i}{2}$, we have

$$
|\Phi(i, 2 k)|=i N_{i} \sum_{s_{0}+\ldots+s_{i}=k}\left(b_{c, 2 s_{i}}+b_{d, 2 s_{i}}\right) \prod_{j=0}^{\frac{i}{2}-1} t_{d, 2 s_{2 j}} t_{c, 2 s_{2 j+1}} .
$$

From [1], we observe that $a_{c, 2 l}=c t_{d, 2 l-2}$, and $a_{d, 2 l}=d t_{c, 2 l-2}$ for $l<g$. Thus, we can rewrite the equation of Corollary 3.8 by using just $a_{c, 2 j}, b_{c, 2 j}, a_{d, 2 j}$ and $b_{d, 2 j}$, for $1 \leq j \leq k$.

\section{Closed walks in $(c, d)$-Regular graphs with initial Vertex out of CYCLE}

In this section, we investigate on the size of $\Lambda(i, 2 k)$. In the following definition, we classify the CWDCC's of $\mathcal{C}$ with initial vertex out of cycle.

Definition 4.1. Let $l$ and $j$ be integers with $1 \leq l \leq k$ and $0 \leq j<i$. Then $\mathfrak{W}\left(v_{j}, l\right)$ is defined as the set of the CWDCCs with cycle $\mathcal{C}$ and initial vertex $z$ such that satisfy in the following conditions:

(i) $z \in V(G-\mathcal{C})$.

(ii) $d\left(z, v_{j}\right)=l$.

(iii) If $\mathcal{W} \in \mathfrak{W}\left(v_{j}, l\right)$, then the first vertex of $\mathcal{C}$ that appears in the walk sequence of $\mathcal{W}$ is $v_{j}$.

The set of initial vertices of $\mathfrak{W}\left(v_{j}, l\right)$ is denoted by $N_{l}\left(v_{j}\right)$, where $1 \leq l \leq k$. It is not difficult to see that if $d\left(v_{j}\right)=c$, then $\left|N_{l}\left(v_{j}\right)\right|$ is equal to:

$$
(c-2)(d-1)^{\left\lceil\frac{l-1}{2}\right\rceil}(c-1)^{\left\lfloor\frac{l-1}{2}\right\rfloor} .
$$

Otherwise,

$$
(d-2)(c-1)^{\left\lceil\frac{l-1}{2}\right\rceil}(d-1)^{\left\lfloor\frac{l-1}{2}\right\rfloor} .
$$

First, we find the number of walks $\mathcal{W} \in \Lambda(i, 2 k)$ with cycle $\mathcal{C}$ such that $v_{0}$ is the first vertex of $\mathcal{C}$ appears in the walk sequence $\mathcal{W}$. For $z_{0} \in N_{l}\left(v_{0}\right)$, since there is not a cycle of length $2 l$, we have a unique path of length $l$ between $z_{0}$ and $v_{0}$, 
say $P_{z_{0} v_{0}}$. We denote this unique path by $z_{0} \sim z_{1} \sim z_{2} \ldots z_{l-1} \sim v_{0}$ (see Fig. 2 ). The set of walks of $\mathfrak{W}\left(v_{0}, l\right)$ with initial vertex $z_{0}$ is denoted by $\Lambda_{v_{0}}^{z_{0}}(l)$.

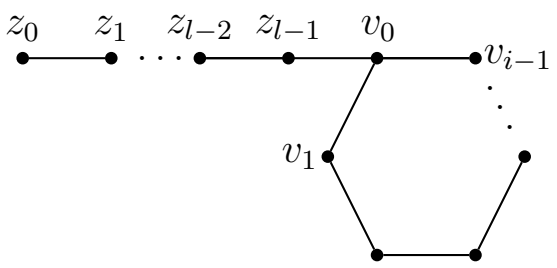

Fig. 2.

Remark 4.2. Suppose that $\mathcal{W} \in \Lambda_{v_{0}}^{z_{0}}(l)$. Denote the initial and terminal vertex of $\mathcal{W}$ by $u_{0}$ and $u_{i+2 l+1}$, respectively. Denote the second vertex of $\mathcal{W}^{* *}$ that appears in $\mathcal{W}$ after the $u_{0}$ by $u_{1}$, and the third vertex of $\mathcal{W}^{* *}$ which appears in $\mathcal{W}$ after the $u_{1}$ by $u_{2}$. Continuing in this way, denote $j$-th vertex of $\mathcal{W}^{* *}$ that appears after $u_{j-2}$, by $u_{j-1}$, for $2 \leq j \leq i+2 l+1$. Now, define the subwalks of $\mathcal{W}$ as follow. For each $j, 0 \leq j \leq i+2 l$, define subwalk $\mathcal{W}_{j}$ of $\mathcal{W}$ with initial and terminal vertex $u_{j}$ and $u_{j+1}$. Hence, we can represent $\mathcal{W}$ uniquely as bellow:

$$
\mathcal{W}=\mathcal{W}_{0} \cdots \mathcal{W}_{i+2 l} \text {. }
$$

Similar to the Remark 2.1, assume that $2 s_{i+2 l}^{\prime}=\ell\left(\mathcal{W}_{i+2 l}\right)$, and $2 s_{j}^{\prime}=\ell\left(\mathcal{W}_{j}\right)-1$, for each $j$ with $0 \leq j \leq i+2 l-1$. By simple computing, we have

$$
\begin{aligned}
s_{0}^{\prime}+\ldots+s_{i+2 l}^{\prime}= & \frac{1}{2}\left(\sum_{j=0}^{i+2 l} \ell\left(\mathcal{W}_{j}\right)-\sum_{j=0}^{i+2 l-1} 1\right) \\
& =\frac{1}{2}(\ell(\mathcal{W})-(i+2 l)) \\
= & \frac{1}{2}((i+2 k)-(i+2 l))=k-l .
\end{aligned}
$$

Definition 4.3. We denote $\Lambda_{v_{0}}^{z_{0}}\left(2 s_{0}, \ldots, 2 s_{i+2 l}\right)$ for the walks of $\mathcal{W} \in \Lambda_{v_{0}}^{z_{0}}(l)$ such that if we represent $\mathcal{W}$ uniquely as $\mathcal{W}=\mathcal{W}_{0} \mathcal{W}_{1} \cdots \mathcal{W}_{i+2 l}$, then $2 s_{j}=\ell\left(\mathcal{W}_{j}\right)-1$, for $0 \leq j<i+2 l$, and $2 s_{i+2 l}=\ell\left(\mathcal{W}_{i+2 l}\right)$.

Since for each walk of $\Lambda_{v_{0}}^{z_{0}}(l)$, there is a unique representation, by Remark 4.2, we have the following result.

Corollary 4.4. For a positive integer $l$ with $1 \leq l \leq k$, we have

$$
\Lambda_{v_{0}}^{z_{0}}(l)=\bigcup_{s_{0}+\ldots+s_{i+2 l}=k-l} \Lambda_{v_{0}}^{z_{0}}\left(2 s_{0}, \ldots, 2 s_{i+2 l}\right) .
$$

Moreover,

$$
\left|\Lambda_{v_{0}}^{z_{0}}(l)\right|=\sum_{s_{0}+\ldots+s_{i+2 l}=k-l}\left|\Lambda_{v_{0}}^{z_{0}}\left(2 s_{0}, \ldots, 2 s_{i+2 l}\right)\right| .
$$


Now, we can state the following lemma.

Lemma 4.5. For $s_{0}+\ldots+s_{i+2 l}=k-l$. If $l$ is even or odd, then we have

$$
\left|\Lambda_{v_{0}}^{z_{0}}\left(2 s_{0}, \ldots, 2 s_{i+2 l}\right)\right|=b_{c, 2 s_{i+2 l}} \prod_{j=0}^{\frac{i}{2}+l-1} t_{c, 2 s_{2 j}} t_{d, 2 s_{2 j+1}}
$$

and

$$
\left|\Lambda_{v_{0}}^{z_{0}}\left(2 s_{0}, \ldots, 2 s_{i+2 l}\right)\right|=b_{d, 2 s_{i+2 l}} \prod_{j=0}^{\frac{i}{2}+l-1} t_{d, 2 s_{2 j}} t_{c, 2 s_{2 j+1}}
$$

respectively.

Proof. Let $\mathcal{W} \in \Lambda_{v_{0}}^{z_{0}}\left(2 s_{0}, \ldots, 2 s_{i+2 l}\right)$. Then Remark 4.2 implies that there are the unique subwalks $\mathcal{W}_{j}$ 's such that $\mathcal{W}=\mathcal{W}_{1} \cdots \mathcal{W}_{i+2 l}$ and $2 s_{j}=\ell\left(\mathcal{W}_{j}\right)-1$, for $0 \leq j<i+2 l$ and $2 s_{i+2 l}=\ell\left(\mathcal{W}_{i+2 l}\right)$. Hence, we can deduce that for $0 \leq j<i+2 l$, $2 s_{j}$ is the length of backward closed cycle-free walk respect to the $\mathcal{W}^{* *}$ with $(j+1)$ th vertex of $\mathcal{W}^{* *}$ as initial. Hence, the number of cycle-free walks in this case is equal to $t_{c, 2 s_{j}}$ or $t_{d, 2 s_{j}}$, depends on the degree initial vertex is $c$ or $d$, respectively. In addition, since $2 s_{i+2 l}=\ell\left(\mathcal{W}_{i+2 l}\right)$, we conclude that $2 s_{i+2 l}$ is the length of closed cycle-free walk with initial vertex $z_{0}$. Therefore, the number of cycle-free walks in this case is $b_{c, 2 s_{i+2 l}}$ or $b_{d, 2 s_{i+2 l}}$, if $l$ is even or odd, respectively. Now, it is enough to show that the walks which computed in the right side of the equation are elements of $\Lambda_{v_{0}}^{z_{0}}\left(2 s_{0}, \ldots, 2 s_{i+2 l}\right)$. Since the initial vertex of the walks is $z_{0}$, we just check that $v_{0}$ is the first vertex of $\mathcal{C}$ that appears in our enumeration. By contradiction assume that $\mathcal{W}^{\prime} \in \Lambda_{v_{0}}^{z_{0}}\left(2 s_{0}, \ldots, 2 s_{i+2 l}\right)$ and vertex $v_{j}, 0<j \leq i-1$, appears before the $v_{0}$ in $\mathcal{W}^{\prime}$. Since $d\left(z_{0}, v_{0}\right)=l$, we have $d\left(z_{0}, v_{j}\right) \leq k-l$. Hence, there is a new cycle of length at most

$$
d\left(z_{0}, v_{0}\right)+d\left(z_{0}, v_{j}\right)+d\left(v_{0}, v_{j}\right) \leq l+(k-l)+\frac{i}{2} .
$$

Since $i+2 k<2 g$, the length of cycle is less than $g$, which is a contradiction.

Lemma 4.6. Let $l$ be a positive integer with $1 \leq l \leq k$ and $z_{0} \in N_{l}\left(v_{0}\right)$. Then

$$
\left|\mathfrak{W}\left(v_{0}, l\right)\right|=\left|N_{l}\left(v_{0}\right)\right|\left|\Lambda_{v_{0}}^{z_{0}}(l)\right|
$$

Proof. Suppose that $w_{0} \in N_{l}\left(v_{0}\right)$ and $w_{0} \neq z_{0}$. Since $d\left(w_{0}\right)=d\left(z_{0}\right), d\left(w_{0}, v_{0}\right)=$ $d\left(z_{0}, v_{0}\right)=l$ and $\Lambda_{v_{0}}^{z_{0}}(l) \cap \Lambda_{v_{0}}^{w_{0}}(l)=\emptyset$, we observe that $\left|\Lambda_{v_{0}}^{z_{0}}(l)\right|=\left|\Lambda_{v_{0}}^{w_{0}}(l)\right|$. Hence, we only compute $\left|\Lambda_{v_{0}}^{z_{0}}(l)\right|$ and finally multiply by $\left|N_{l}\left(v_{0}\right)\right|$.

Lemma 4.7. The number of walks $\mathcal{W} \in \Lambda(i, 2 k)$ with cycle $\mathcal{C}$ such that $v_{0}$ is the first vertex of $\mathcal{C}$ that appears in $\mathcal{W}$ is

$$
\sum_{l=1}^{k}\left|\mathfrak{W}\left(v_{0}, l\right)\right|
$$

Proof. Let $\mathcal{W} \in \Lambda(i, 2 k)$ with cycle $\mathcal{C}$ and initial vertex $z_{0}$ such that the first vertex of $\mathcal{C}$ that appears in $\mathcal{W}$ is $v_{0}$. In this case, $1 \leq d\left(z_{0}, v_{0}\right) \leq k$. Hence $\mathcal{W} \in \mathfrak{W}\left(v_{0}, l\right)$, where $l=d\left(z_{0}, v_{0}\right)$. Since $\mathfrak{W}\left(v_{0}, l\right) \cap \mathfrak{W}\left(v_{0}, l^{\prime}\right)=\emptyset$, for distinct $l$ and $l^{\prime}$, the assertion holds. 
Note that we have $\frac{i}{2}$ vertices of degree $c$ and $\frac{i}{2}$ vertices of degree $d$ in $\mathcal{C}$. In addition, for $0 \leq j \leq \frac{i}{2}-1$, we have

$$
\left|\mathfrak{W}\left(v_{0}, l\right)\right|=\left|\mathfrak{W}\left(v_{2 j}, l\right)\right|,
$$

and

$$
\left|\mathfrak{W}\left(v_{1}, l\right)\right|=\left|\mathfrak{W}\left(v_{2 j+1}, l\right)\right| .
$$

Hence, we have the following consequence.

Corollary 4.8. For a positive integer $k$ with $1 \leq k<g-\frac{i}{2}$, we have

$$
|\Lambda(i, 2 k)|=i N_{i} \sum_{l=1}^{k}\left(\left|\mathfrak{W}\left(v_{0}, l\right)\right|+\left|\mathfrak{W}\left(v_{1}, l\right)\right|\right) .
$$

Remark 4.9. It is not difficult to see that to find $|\Lambda(i, 2 k)|$, we may only calculate $\left|\mathfrak{W}\left(v_{0}, l\right)\right|$ for $1 \leq l \leq k$. Because $\mathcal{B}_{c, d}$ is a bi-regular graph and we have similar result for $\left|\mathfrak{W}\left(v_{1}, l\right)\right|$. Since we know the value $\left|N_{l}\left(v_{0}\right)\right|$, it is enough to check the $\left|\Lambda_{v_{0}}^{z_{0}}(l)\right|$ to find $\left|\mathfrak{W}\left(v_{0}, l\right)\right|$ for $1 \leq l \leq k$.

For finding the number of cycles of length $i$ in $\mathcal{B}_{c, d}$, it is enough to investigate the value $\Psi_{i}\left(c, d, \mathcal{B}_{c, d}\right)$, by Theorem 2.3. In the next two theorems we enumerate $\Psi_{j}\left(c, d, \mathcal{B}_{c, d}\right)$ for $j=g+2, g+4$. Our proof is simpler than the proof of Theorem 2 and Theorem 3 in [4]. Finally, we compute $\Psi_{g+6}\left(c, d, \mathcal{B}_{c, d}\right)$.

Theorem 4.10. Let $G$ be a $(c, d)$-regular graph. Then

$$
\Psi_{g+2}\left(c, d, \mathcal{B}_{c, d}\right)=g N_{g}(g+2)(c+d-2) .
$$

Proof. To compute $\Psi_{g+2}\left(c, d, \mathcal{B}_{c, d}\right)$, we calculate the values $|\Phi(g, 2 k)|$ and $|\Lambda(g, 2 k)|$, respectively. Since $k=1$, Corollary 3.8 implies that

$$
|\Phi(g, 2)|=g N_{g} \sum_{s_{0}+\ldots+s_{g}=1}\left(b_{c, 2 s_{g}}+b_{d, 2 s_{g}}\right) \prod_{j=0}^{\frac{g}{2}-1} t_{d, 2 s_{2 j}} t_{c, 2 s_{2 j+1}} .
$$

In this case, we have $g+1$ cases for $\left(s_{0}, \ldots, s_{g}\right)$. If $s_{g}=0$, then $b_{c, 0}+b_{d, 0}=2$. Since there are $\frac{g}{2}$ vertices of degree $c$ and $\frac{g}{2}$ vertices of degree $d$, the number of CWDCCs in this case is $2 \frac{g}{2} t_{c, 2}+2 \frac{g}{2} t_{d, 2}$. If $s_{g}=1$, then the number of CWDCCs is $b_{c, 2}+b_{d, 2}$. Therefore,

$$
|\Phi(g, 2)|=g N_{g}\left(g\left(t_{c, 2}+t_{d, 2}\right)+b_{c, 2}+b_{d, 2}\right) .
$$

By the corollary 4.8, we have

$$
\begin{aligned}
|\Lambda(g, 2)| & =g N_{g} \sum_{l=1}^{1}\left(\left|\mathfrak{W}\left(v_{0}, l\right)\right|+\left|\mathfrak{W}\left(v_{1}, l\right)\right|\right) \\
& =g N_{g}\left(\left|\mathfrak{W}\left(v_{0}, 1\right)\right|+\left|\mathfrak{W}\left(v_{1}, 1\right)\right|\right) .
\end{aligned}
$$

It is enough to find the value $\left|\Lambda_{v_{0}}^{z_{0}}(1)\right|$, by Remark 4.9. So

$$
\left|\Lambda_{v_{0}}^{z_{0}}(1)\right|=\sum_{s_{0}+\ldots+s_{g+2}=0}\left|\Lambda_{v_{0}}^{z_{0}}\left(2 s_{0}, \ldots, 2 s_{g+2}\right)\right|=1
$$


Therefore, we deduce that $\left|\mathfrak{W}\left(v_{0}, 1\right)\right|=\left|N_{1}\left(v_{0}\right)\right|$ and $\left|\mathfrak{W}\left(v_{1}, 1\right)\right|=\left|N_{1}\left(v_{1}\right)\right|$. Thus,

$$
|\Lambda(g, 2)|=g N_{g}\left(\left|N_{1}\left(v_{0}\right)\right|+\left|N_{1}\left(v_{1}\right)\right|\right) . \quad(* *)
$$

By the equations $(*)$ and $(* *)$ we conclude that

$$
\Psi_{g+2}\left(c, d, \mathcal{B}_{c, d}\right)=g N_{g}\left(g\left(t_{c, 2}+t_{d, 2}\right)+\left(b_{c, 2}+b_{d, 2}\right)+\left(\left|N_{1}\left(v_{0}\right)\right|+\left|N_{1}\left(v_{1}\right)\right|\right)\right) .
$$

Theorem 4.11. Let $G$ be a $(c, d)$-regular graph. Then

$$
\begin{aligned}
\Psi_{g+4}\left(c, d, \mathcal{B}_{c, d}\right) & =(g+2) N_{g+2}(g+4)(c+d-2) \\
& +g N_{g}\left[g(c+d-2)^{2}+(c+d)(c+d-1)\right] \\
& +g N_{g}\left(2\left(\begin{array}{c}
\frac{g}{2} \\
2
\end{array}\right)\left[(c-1)^{2}+(d-1)^{2}\right]+2\left(\frac{g}{2}\right)^{2}(c-1)(d-1)\right) \\
& +g N_{g} \frac{g}{2}[(c+d)(c+d-2)] \\
& +g N_{g}\left(\left(\frac{g}{2}+1\right)(c+d-2)(c+d-4)+(c-2)(2 d-1)+(d-2)(2 c-1)\right) .
\end{aligned}
$$

Proof. To compute the number of CWWCs of the length $g+4$, we need to know the number of cycles of length $g$ and $g+2$ which have already enumerated. Thus, we arise the following two cases:

Case 1. The closed walk of length $g+4$ contains a cycle of length $g$. We first compute $|\Phi(g, 4)|$. In this case, $k=2$ and Corollary 3.8 implies that

$$
|\Phi(g, 4)|=g N_{g} \sum_{s_{0}+\ldots+s_{g}=2}\left(b_{c, 2 s_{g}}+b_{d, 2 s_{g}}\right) \prod_{j=0}^{\frac{g}{2}-1} t_{d, 2 s_{2 j}} t_{c, 2 s_{2 j+1}} .
$$

Depending on $s_{j}$ is one or two, we consider the following subcases:

Case 1.1. Each $s_{j}$ is two or zero. In this case, if $s_{g}$ is zero or not, then the number of closed CWDCCs is equal to

$$
2 \frac{g}{2} t_{c, 4}+2 \frac{g}{2} t_{d, 4}
$$

or

$$
b_{c, 4}+b_{d, 4}
$$

respectively.

Case 1.2. Each $s_{j}$ is one or zero. Suppose that $s_{g}=0$. In this sense, there are three cases to select two ones for $s_{j}$ 's. If both vertices have the same degree, then the number of CWDCCs is

$$
2\left(\begin{array}{c}
\frac{g}{2} \\
2
\end{array}\right)\left[t_{c, 2}^{2}+t_{d, 2}^{2}\right]
$$

If the degree of vertices are different, then the number of CWDCCs is

$$
2\left(\frac{g}{2}\right)^{2} t_{c, 2} t_{d, 2}
$$


Now, suppose that $s_{g} \neq 0$. In this case, $s_{g}=1$ and so there is another $j^{\prime}$ such that $0 \leq j^{\prime}<g$ and $s_{j}^{\prime}=1$. Hence, the number of CWDCCs is

$$
\frac{g}{2}\left(b_{c, 2}+b_{d, 2}\right)\left(t_{c, 2}+t_{d, 2}\right) \text {. }
$$

Thus,

$$
\begin{aligned}
|\Phi(g, 4)| & =g N_{g}\left(g\left(t_{c, 4}+t_{d, 4}\right)+\left(b_{c, 4}+b_{d, 4}\right)+2\left(\begin{array}{c}
\frac{g}{2} \\
2
\end{array}\right)\left[t_{c, 2}^{2}+t_{d, 2}^{2}\right]\right. \\
& \left.+2\left(\frac{g}{2}\right)^{2} t_{c, 2} t_{d, 2}+\frac{g}{2}\left(b_{c, 2}+b_{d, 2}\right)\left(t_{c, 2}+t_{d, 2}\right)\right) .
\end{aligned}
$$

Now, we want to find $|\Lambda(g, 4)|$. By the Corollary 4.8, we have

$$
|\Lambda(g, 4)|=g N_{g} \sum_{l=1}^{2}\left(\left|\mathfrak{W}\left(v_{0}, l\right)\right|+\left|\mathfrak{W}\left(v_{1}, l\right)\right|\right)
$$

It is enough to find the values $\left|\Lambda_{v_{0}}^{z_{0}}(1)\right|$ and $\left|\Lambda_{v_{0}}^{z_{0}}(2)\right|$, by Remark 4.9, First consider $l=1$ and Lemma 4.5 implies that

$$
\begin{aligned}
\left|\Lambda_{v_{0}}^{z_{0}}(1)\right| & =\sum_{s_{0}+\ldots+s_{g+2}=1}\left|\Lambda_{v_{0}}^{z_{0}}\left(2 s_{0}, \ldots, 2 s_{g+2}\right)\right| \\
& =\left(\frac{g}{2}+1\right) t_{c, 2}+\left(\frac{g}{2}+1\right) t_{d, 2}+b_{d, 2} .
\end{aligned}
$$

Since $\left|\Lambda_{v_{0}}^{z_{0}}(2)\right|=1$, we have

$$
\begin{aligned}
|\Lambda(g, 4)| & =g N_{g}\left(\left(\frac{g}{2}+1\right)\left(\left|N_{1}\left(v_{0}\right)\right|+\left|N_{1}\left(v_{1}\right)\right|\right)\left(t_{c, 2}+t_{d, 2}\right)\right. \\
& \left.+\left|N_{1}\left(v_{0}\right)\right| b_{d, 2}+\left|N_{1}\left(v_{1}\right)\right| b_{c, 2}+N_{2}\left(v_{0}\right)+N_{2}\left(v_{1}\right)\right) .
\end{aligned}
$$

Case 2. The closed walk of length $g+4$ that contains a cycle of length $g+2$. From the proof of Theorem 4.10, the number of CWDCCs in this case is

$$
(g+2) N_{g+2}\left((g+2)\left(t_{c, 2}+t_{d, 2}\right)+\left(b_{c, 2}+b_{d, 2}\right)+\left(\left|N_{1}\left(v_{0}\right)\right|+\left|N_{1}\left(v_{1}\right)\right|\right)\right) .
$$

Now, we find the value $\Psi_{g+6}\left(c, d, \mathcal{B}_{c, d}\right)$ in the following three lemmas.

Lemma 4.12. Let $G$ be a $(c, d)$-regular graph. Then we have

$$
\begin{aligned}
|\Phi(g, 6)| & =g N_{g}\left(\left((c-1)^{2}+(d-1)^{2}\right)(c+d-2)\left[g+2\left(\begin{array}{c}
\frac{g}{2} \\
2
\end{array}\right)+2\left(\begin{array}{c}
\frac{g}{2} \\
3
\end{array}\right)\right]\right. \\
& +\left((c-1)^{2}+(d-1)^{2}\right)(c+d)\left[\left(\begin{array}{c}
\frac{g}{2} \\
2
\end{array}\right)+1\right] \\
& +(c-1)(d-1)(c+d-2)\left[3 g+g^{2}+g\left(\begin{array}{c}
\frac{g}{2} \\
2
\end{array}\right)-2\left(\begin{array}{c}
\frac{g}{2} \\
3
\end{array}\right)\right] \\
& \left.+(c+d)\left[(3 c d-c-d)+\frac{g^{2}}{4}(c-1)(d-1)\right]\right)
\end{aligned}
$$


and

$$
\begin{aligned}
|\Lambda(g, 6)| & =g N_{g}\left(( c + d - 4 ) \left[\left(\frac{g}{2}+1\right)(c+d-2)^{2}+\left(\left(\frac{g}{2}+1\right)^{2}+1\right)(c-1)(d-1)\right.\right. \\
& \left.+\left(\begin{array}{c}
\frac{g}{2}+1 \\
2
\end{array}\right)\left((c-1)^{2}+(d-1)^{2}\right)\right] \\
& +(2 c d-2 c-2 d)\left[(c+d-1)+\left(\frac{g}{2}+1\right)(c+d-2)\right] \\
& +\left(\frac{g}{2}+2\right)(c+d-2)[(c-2)(d-1)+(d-2)(c-1)] \\
& +c(c-2)(d-1)+d(d-2)(c-1)) .
\end{aligned}
$$

Proof. To enumerate the number of CWDCCs in this case, we first investigate $|\Phi(g, 6)|$. From the Corollary 3.8, we have

$$
|\Phi(g, 6)|=g N_{g} \sum_{s_{0}+\ldots+s_{g}=3}\left(b_{c, 2 s_{g}}+b_{d, 2 s_{g}}\right) \prod_{j=0}^{\frac{g}{2}-1} t_{d, 2 s_{2 j}} t_{c, 2 s_{2 j+1}} .
$$

In our proof, we avoid using $g N_{g}$ in our calculating. Now, consider the following three subcases:

Case 1. Each $s_{j}$ is zero or three. In the above summation, if $s_{g}=0$, then the number of CWDCCs is

$$
2 \frac{g}{2} t_{c, 6}+2 \frac{g}{2} t_{d, 6}
$$

If $s_{g}=3$, then the number of CWDCCs in this case is equal to:

$$
b_{c, 6}+b_{d, 6} \text {. }
$$

Case 2. Each $s_{j}$ is zero, one, or two. Depending on whether $s_{g}$ is zero or not, the number of CWDCCs is

$$
2\left(\begin{array}{c}
\frac{g}{2} \\
2
\end{array}\right)\left(t_{c, 2} t_{c, 4}+t_{d, 2} t_{d, 4}\right)+2 \frac{g^{2}}{4}\left(t_{c, 2} t_{d, 4}+t_{c, 4} t_{d, 2}\right),
$$

and

$$
\frac{g}{2}\left(\left(b_{c, 4}+b_{d, 4}\right)\left(t_{c, 2}+t_{d, 2}\right)+\left(b_{c, 2}+b_{d, 2}\right)\left(t_{c, 4}+t_{d, 4}\right)\right)
$$

respectively.

Case 3. Each $s_{j}$ is zero or one. Again, suppose that $s_{g}=0$, then we have three ones in distinct $s_{j}$ 's. Hence, the number of CWDCCs is equal to

$$
2\left(\begin{array}{c}
\frac{g}{2} \\
3
\end{array}\right)\left(t_{c, 2}^{3}+t_{d, 2}^{3}\right)+2\left(\begin{array}{c}
\frac{g}{2} \\
2
\end{array}\right)\left(\begin{array}{c}
\frac{g}{2} \\
1
\end{array}\right)\left(t_{c, 2}^{2} t_{d, 2}+t_{c, 2} t_{d, 2}^{2}\right) .
$$

If $s_{g} \neq 0$, then $s_{g}=1$ and the number of CWDCCs is

$$
\left(b_{c, 2}+b_{d, 2}\right)\left(\left(\begin{array}{c}
\frac{g}{2} \\
2
\end{array}\right)\left(t_{c, 2}^{2}+t_{d, 2}^{2}\right)+\left(\frac{g}{2}\right)^{2} t_{c, 2} t_{d, 2}\right) .
$$


To complete the proof in this case, we find the value $|\Lambda(g, 6)|$. By the corollary 4.8, we have

$$
|\Lambda(g, 6)|=g N_{g} \sum_{l=1}^{3}\left(\left|\mathfrak{W}\left(v_{0}, l\right)\right|+\left|\mathfrak{W}\left(v_{1}, l\right)\right|\right) .
$$

It is enough to find $\left|\Lambda_{v_{0}}^{z_{0}}(l)\right|$ for $1 \leq l \leq 3$. Hence, we consider the following three subcases:

Case a. Suppose that $l=1$. Since $l$ is odd, Corollary 4.4 and Lemma 4.5 imply that

$$
\left|\Lambda_{v_{0}}^{z_{0}}(1)\right|=\sum_{s_{0}+\ldots+s_{g+2}=2} b_{d, 2 s_{g+2}} \prod_{j=0}^{\frac{g}{2}} t_{d, 2 s_{2 j}} t_{c, 2 s_{2 j+1}} .
$$

If $s_{j} \in\{0,2\}$, then the number of CWDCCs in this case is equal to:

$$
\left(\frac{g}{2}+1\right)\left(t_{c, 4}+t_{d, 4}\right)+b_{d, 4}
$$

Now, suppose that $s_{j} \in\{0,1\}$. Depending on whether $s_{g+2}$ is zero or not, the number of CWDCC's is

$$
\left(\begin{array}{c}
\frac{g}{2}+1 \\
2
\end{array}\right)\left(t_{c, 2}^{2}+t_{d, 2}^{2}\right)+\left(\frac{g}{2}+1\right)^{2} t_{c, 2} t_{d, 2}
$$

and

$$
\left(\frac{g}{2}+1\right) b_{d, 2}\left(t_{c, 2}+t_{d, 2}\right)
$$

respectively.

Case b. Suppose that $l=2$. Since $l$ is even, we have

$$
\left|\Lambda_{v_{0}}^{z_{0}}(2)\right|=\sum_{s_{0}+\ldots+s_{g+4}=1} b_{c, 2 s_{g+4}} \prod_{j=0}^{\frac{g}{2}+1} t_{c, 2 s_{2 j}} t_{d, 2 s_{2 j+1}} .
$$

Depending on $s_{j}$ is zero or not, we have the following number as the CWDCCs.

$$
\left(\frac{g}{2}+2\right)\left(t_{c, 2}+t_{d, 2}\right)+b_{c, 2}
$$

Case c. Assume that $l=3$. Therefore, by Corollary 4.4 and Lemma 4.5 we have

$$
\left|\Lambda_{v_{0}}^{z_{0}}(3)\right|=\sum_{s_{0}+\ldots+s_{g+6}=0} b_{d, 2 s_{g+6}} \prod_{j=0}^{\frac{g}{2}+2} t_{d, 2 s_{2 j}} t_{c, 2 s_{2 j+1}}=1 .
$$

Lemma 4.13. Let $G$ be a $(c, d)$-regular graph. Then the number of $C W W C$ s of length $g+6$ with cycle of length $g+2$ is equal to

$$
\begin{aligned}
& (g+2) N_{g+2}\left[(g+2)(c+d-2)^{2}+(c+d)(c+d-1)\right] \\
& +(g+2) N_{g+2}\left(2\left(\begin{array}{c}
\frac{g+2}{2} \\
2
\end{array}\right)\left[(c-1)^{2}+(d-1)^{2}\right]+2\left(\frac{g+2}{2}\right)^{2}(c-1)(d-1)\right) \\
& +(g+2)\left(\frac{g+2}{2}\right) N_{g+2}[(c+d)(c+d-2)]
\end{aligned}
$$




$$
+(g+2) N_{g+2}\left(\left(\frac{g+2}{2}+1\right)(c+d-2)(c+d-4)+(c-2)(2 d-1)+(d-2)(2 c-1)\right) .
$$

Proof. We already computed the values of $|\Phi(g, 4)|$ and $|\Lambda(g, 4)|$ in the case 1 of the proof of Theorem 4.11. Hence, we have

$$
\begin{aligned}
|\Phi(g+2,4)| & =(g+2) N_{g+2}\left((g+2)\left(t_{c, 4}+t_{d, 4}\right)+\left(b_{c, 4}+b_{d, 4}\right)+2\left(\begin{array}{c}
\frac{g+2}{2} \\
2
\end{array}\right)\left[t_{c, 2}^{2}+t_{d, 2}^{2}\right]\right. \\
& \left.+2\left(\frac{g+2}{2}\right)^{2} t_{c, 2} t_{d, 2}+\frac{g+2}{2}\left(b_{c, 2}+b_{d, 2}\right)\left(t_{c, 2}+t_{d, 2}\right)\right)
\end{aligned}
$$

and

$$
\begin{aligned}
|\Lambda(g+2,4)| & =(g+2) N_{g+2}\left(\left(\frac{g+2}{2}+1\right)\left(\left|N_{1}\left(v_{0}\right)\right|+\left|N_{1}\left(v_{1}\right)\right|\right)\left(t_{c, 2}+t_{d, 2}\right)\right. \\
& \left.+\left|N_{1}\left(v_{0}\right)\right| b_{d, 2}+\left|N_{1}\left(v_{1}\right)\right| b_{c, 2}+N_{2}\left(v_{0}\right)+N_{2}\left(v_{1}\right)\right) .
\end{aligned}
$$

Lemma 4.14. Let $G$ be a $(c, d)$-regular graph. Then the number of $C W W C$ s of length $g+6$ with cycle of length $g+4$ is equal to

$$
(g+4) N_{g+4}(g+6)(c+d-2) .
$$

Proof. Since the values $|\Phi(g, 2)|$ and $|\Lambda(g, 2)|$ are known by the proof of Theorem 4.10. Thus,

$$
|\Phi(g+4,2)|=(g+4) N_{g+4}\left((g+4)\left(t_{c, 2}+t_{d, 2}\right)+b_{c, 2}+b_{d, 2}\right),
$$

and

$$
|\Lambda(g+4,2)|=(g+4) N_{g+4}\left(\left|N_{1}\left(v_{0}\right)\right|+\left|N_{1}\left(v_{1}\right)\right|\right) .
$$

\section{REFERENCES}

[1] I. Blake And S. Lin, On Short Cycle Enumeration in Biregular Bipartite Graphs, IEEE Trans. Inf. Theory, Dec. 2017, available online at: http://ieeexplore.ieee.org/document/8225637/.

[2] K. M. Chugg, A. Anastasopoulos, and X. Chen, Iterative Detection: Adaptivity, Complexity Reduction, and Applications. Norwell, MA: Kluwer, 2001.

[3] D. Cvetkovic, P. Rowlinson, and S. Simic, An introduction to the theory of graph spectra, London Mathematical Society, Student Texts, vol. 75, 2010.

[4] A. Dehghan And A. Banihashemi, On Computing the Multiplicity of Short Cycles in Bipartite Graphs Using the Degree Distribution and the Spectrum of the Graph, arXiv preprint arXiv:1806.01433, Jun. 2018.

[5] A. Dehghan And A. Banihashemi, On the Tanner Graph Cycle Distribution of Random LDPC, Random Protograph-Based LDPC, and Random Quasi-Cyclic LDPC Code Ensembles, IEEE Trans. Inf. Theory, vol. 64, no. 6, pp. 4438-4451, June 2018.

[6] J. Flum And M. Grohe, The parameterized complexity of counting problems, SIAM J. Comput., vol. 33, no. 4, pp. 892-922, 2004.

[7] T. R. Halford and K. M. Chugg, An algorithm for counting short cycles in bipartite graphs, IEEE Trans. Inform. Theory, vol. 52, no. 1, pp. 287-292, Jan. 2006.

[8] X.-Y. Hu, E. Eleftheriou, And D. M. Arnold, Regular and irregular progressive edge-growth

Tanner graphs, IEEE Trans. Inform. Theory, vol. 51, no. 1, pp. 386-398, Jan. 2005. 
[9] M. Karimi And A. H. Banihashemi, On the girth of quasi-cyclic protograph LDPC codes, IEEE Trans. Inform. Theory, vol. 59, no. 7, pp. 4542-4552, July 2013.

[10] M. Karimi and A. H. Banihashemi, Message-passing algorithms for counting short cycles in a graph, IEEE Trans. Communications, vol. 61, no. 2, pp. 485-495, Feb. 2013.

[11] Y. Mao And A. H. Banihashemi, A heuristic search for good low-density parity-check codes at short block lengths, in Proc. Int. Conf. Communications, vol. 1, Helsinki, Finland, Jun. 2001, pp. 41-44.

[12] T. Richardson, M. A. Shokrollahi, and R. Urbanke, Design of capacity approaching irregular low-density parity check codes, IEEE Trans. Inform. Theory, vol. 47, no. 2, pp. 619-637, Feb. 2001.

[13] R. M. Tanner, A recursive approach to low-complexity codes, IEEE Trans. Inform. Theory, vol. 27, no. 5, pp. 533-547, Sep 1981.

[14] H. Xiao And A. H. Banihashemi, Error rate estimation of low-density parity-check codes on binary symmetric channels using cycle enumeration, IEEE Trans. Communications, vol. 57, no. 6, pp. 1550-1555, June 2009.

Department of Pure Mathematics, Ferdowsi University, Mashhad, Iran.

E-mail address: mohsenalinejad96@gmail.com

E-mail address: khashyar@ipm.ir 